\title{
Art in the Digital Age: Posthumanism, A.I. and the body
}

\author{
Grayson Aleksandr Mills \\ Graduate Student (Virginia \\ Commonwealth University) \\ 311 W Franklin St APT 409 \\ Richmond VA 23220, USA \\ millsg3@vcu.edu
}

\begin{abstract}
The concept of the body is being explored within art in new ways regarding its digital presentation and non-traditional topics regarding sentience and humanity. Two examples of this are the concepts of online presence and digital personhood. Both of these concepts, which I explore through an art historical perspective, involve considering what makes human experiences, how these experiences can be manipulated, and what the boundaries of the mind and the body are. In this paper I use the works Excellences and Perfections (2014) by Amalia Ulman and Hyperlinks or It Didn't Happen (2014) by Cecile B. Evans to discuss the body in the digital age as a representation of posthumanism. I also place my concepts of post humanism within the existing scholarship to consider the ways in which artificial intelligence and non-traditional human forms can be considered as living.
\end{abstract}

Posthumanism. Artificial intelligence. Web art. Social media. Online presence. Digital personhood. Digital consciousness.

\section{INTRODUCTION}

The 'human experience' is undergoing radical changes as technology advances. What was once understood to be simple facts about physical existence is now being questioned as boundaries are pushed and new capacities of experience are being created. Two examples of this are the concepts of online presence and digital personhood. Both of these concepts, which I will explore in more depth through this paper through an art historical perspective, focus on the phenomenon of experiencing human interactions without being in the same physical space with the one you are interacting with. In this paper I will be using the works Excellences and Perfections (2014) by Amalia Ulman and Hyperlinks or It Didn't Happen (2014) by Cecile B. Evans to discuss the body in the digital age as a representation of posthumanism.

\section{DIGITAL POSTHUMANISM}

To begin I will define my use of terms such as 'digital,' 'body,' 'living,' 'presence,' and 'posthuman' in regards to this particular project. First, I'm using 'digital' to indicate any presence that can be accessed through the Internet or can be experienced solely through a screen or other similar device, such as that of augmented and artificial realities. This definition is meant to work solely with visuals, so concepts such as digital versus analog audio will not be considered in this project. In regards to 'digital' interacting with art, I will refer to Ippolito and Rinehart's (2014) definition from their book Re-collection, which states:

\footnotetext{
'Digital art' refers to artworks that are born digital; they are created and experienced using digital media (they might not rely exclusively on digital media, but they incorporate them in ways that are essential and inextricable).
}

Next, the type of posthumanism I will primarily be working with is an extension of transhuman, as established by Gorreau (2005). That is to say, if transhumanism exists as a transition between human and non-human entities, such as cybernetics, then I propose posthumanism indicates being beyond the human body entirely. I will address the theory of posthumanism that revolves around decentralising humanity, such as the one Wolfe suggests, but I will be doing this exclusively as a case of comparison. My use of 'living' is to be applied to anything that can form its 
own thoughts. This is a controversial definition, but it is inherently meant to include artificial intelligence and other programs that function beyond simple input/output mechanisms.

My concept of 'presence' moves beyond the necessity of space and time. While space and time can be considered abstractly, such as the runtime of a video or the space a string of code takes up on a server, there is inherently a shift away from the necessity to exist in one physical space and time to experience the phenomena I will be discussing. Here, 'presence' will also indicate anything that can be perceived sensually, but I will only be utilising vision and hearing for this purpose. For example, an image indicates the presence of a subject via an image of that subject.

My working definition of 'body' is a physical manifestation in which the operative mind is working. This definition relies on hylomorphism and the Cartesian mind/body divide as a mere framework for the distinction of the mind from the body. Under my operating term, a body need not be flesh and blood as often implied by the term 'body,' but instead includes something like a processor as a form of a body. This, again, is to intentionally include something like a processor as a form of artificial intelligence within the operating definition of 'body' as well as 'living,' considering A.I. in a loose form of non-human computing.

\subsection{Digital personality \& Online Identity}

The creation of a digital personality has become prolific with the advent of social media. People are able to connect with one another from miles away without having ever met in a physical space. With this connection is a presentation of self that is not exclusively dependent on the physical presence of the self. Many people today view their social media presence as an extension of their own being. So much so that to receive 'likes' becomes a form of psychological and material validation (Emmelheinz 2015).

The extension of someone's 'genuine experience' that is represented on their social media accounts can be varied based on someone's preference of presentation. This phenomenon of the 'genuine experience' can be found in the multitude of accounts on a website like Instagram, in which people upload a series of pictures to create an identity that can be accessed exclusively through that channel. It is not necessarily true that any of these represents or does not represent the person's life. Which is to say, the images one has on their social media does not inherently represent one thing or another about a persons life, only that the photos created at some point in time in some location. However, the weight people typically assign to photographs of being a direct representation of a persons life demonstrates a way digital presence is often equated to a physical presence to the point that people can feel like they are familiar with a person based solely on the way they are presented digitally. Furthermore, Instagram presentations become associated with an arbitrary perception of 'realness' or 'fakeness' that ignores aspects of performativity (Butler 1990) that occurs in all presentations of self, physical or digital.

Performativity, as explored by Butler explores the ways in which people are constantly performing within society, whether consciously or subconsciously, to fit into a social category or to work against it, certain acts indicate attributes and information about the performer (i.e. person). This theory can be applied to the way in which people upload, or choose not to upload certain information about themselves and their experiences, and through what lens this information is filtered through.

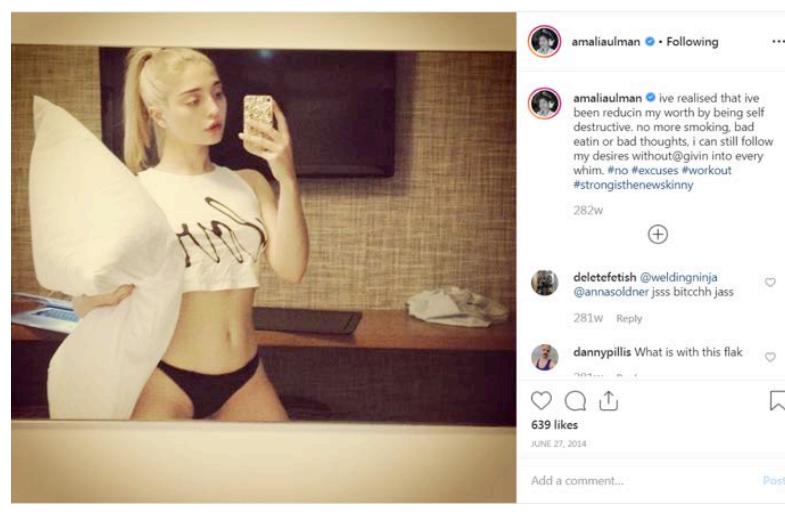

Figure 1: Image from series 'Excellences and Perfections' (2014) from @amaliaulman (Instagram).

\subsection{Excellences and Perfections}

The concept of 'realness' and 'fakeness' on social media and the crafting of a digital personality and online identity is explored in Excellences and Perfections (2014) by Amalia UIman. Excellences and Perfections was released exclusively on Instagram, with the presentation of a series of 172 images and 12 videos from April 2014 to September 2014. The piece was not revealed to be a performance until after it was completed. Upon completion, the series was digitally preserved by Rhizome and the New Museum in New York, and later published as a catalogue with additional accompanying text (adding on to her existing captions) in 2018 (Ulman 2018).

The viewers of the piece follow Ulman as her character moves to Los Angeles, California and experiences the rise and fall of her affluence and mental well-being. The piece begins with Ulman 
posting in a documentary style of a small-town woman becoming a sugar baby who is sharing her daily life via 'selfies' and artistically driven still life's. As the weeks progress, Ulman's character strives for excellence, in the form of beauty, wealthy and status, but never meeting her (or her followers) dreams of perfection for the young woman. The photos move from a colour scheme of whites, pale pinks, and gold, to darker browns, blacks, and greys, as the posts of this character become more self conscious, despondent, and focused on validation from people commenting on her photos.

This visual and implied psychological decay continues to the point that is implied she is having a mental breakdown and is seeking out rehabilitation from the pressure and failure to achieve perfection. This breakdown, as part of the series activates a personal sense of loss or schadenfreude for her followers depending on their perception of Ulman and whether they wish to see her rise or fall. The series concludes as the character returns to posting after two weeks of silence, having previously posted near daily, to apologise for her behaviour and to thank her followers for their support. The 'support' being witnessing the turmoil of the young woman's vulnerability, since the only way these followers could 'support' her fame (and therefore excellence) is by continuing to follow her account throughout the events.

The series continues for one month after that point as viewers follow Ulman's transition from misery as the character attempts to build herself back up, the colour scheme transitioning once more into lighter colours. However, it is clear that the character continues to rely on some sort of feedback from her followers, who replied in the comment section of her images with both strong support commending her perseverance and bravery and strong critique indicating she was foolish for even being on the platform in the first place. With captions such as "thank you followers for being so nice and supportive", it is evident that despite her growth, she continues to value her followers and still seeks to attain some sort of excellence, though the and goal of that excellence remains unclear.

While a dramatic case, this narrative demonstrates the previously mentioned, very real phenomenon Emmelheinz explores, of people valuing likes and online presence as much as any other interpersonal relationship and desires to be accepted by one's peers. With more young people on the Internet this consideration of the effects of likes as validation has become an even greater point of contention to cognitive development than in previous generations (Leventhal 2019). Throughout the piece it was not considered that the entire plot of these images was fabricated. The followers of the account meant to treat Ulman's character as if she were a 'real person.' The revealing of this piece as a performance demonstrates how much people associate online presence with physical presence and online identity as a representation of self. Given that people were often unable to separate Ulman's character from Ulman herself, with comments now present on her work calling her a 'fake.' Alternatively, people valued the representation of Ulman's character so much that she became a reality within the minds of her followers. Regardless, the digital personality Ulman crafted had gained a life of its own that, in some cases, overshadowed the actual life of UIman.

\subsubsection{The Role of Performance Art}

As Rozsa Farkas states in the exhibition catalogue of UIman's piece (UIman 2018):

The notion of the authentic loomed as synthetic as ever, and challenged the terms around which we understand performance art.

The piece directly undermines assumptions we have about performance art, namely their temporality and spatial presence. Ulman's presence exists away from her physical body, taking up a digital presence, pushing the boundaries of both performance art, and the general understanding of the digital personality representing the physical. The personhood of the body is questioned as the viewer is forced to reconcile with the fact that Ulman's character, and Ulman herself are two different realities, each of which demonstrates different properties of humanity and online presence.

\subsubsection{Considering 'Presence'}

The consideration of 'presence' is also called into question. I have used the term multiple times in description of the piece, but the term has its own significance. It is clear that a piece like Ulman's considers the necessity of time and space that is so often connected to performance art. One could consider the 'time' of the piece to be a few months in 2014, and the 'space' could be considered Los Angeles, or perhaps the memory space the Instagram account takes up in the website's server room. But, these formulations of space and time inherently extend beyond the presence that is understood through a piece like Ambromovic's The Artist is Present (2010). Ambromovic's piece which was hyper-fixated on time and space, where the experience of the piece was limited to one space and one time with the profound interactions that came out of the piece being limited to an experience that now only exists in the past. Ulman's work demonstrates the shift in the spaciotemporal reliant concept of presence by working with the sustainability of the online presence. As I mentioned previously, both of these concepts become abstract, as the medium inherently allows 
for the work to be shared at any time and anywhere that someone provided a suitable device.

As it pertains to viewing the work, the only space the work was originally presented in was that of cyberspace. Cyberspace as a malleable form, allows for a certain proximity that cannot be attained in a traditional performance. In a way, Ulman's character was a part of each follower's life as the performance infiltrated their daily Instagram feeds. This aspect of the work being incorporated into people's personal Instagram feed is what connected so many people to the performance as it was occurring. It is also likely part of what caused so much outrage, as a response to finding out the 'person' that thought they knew, was not a physical being at all. Since we can assume her followers made the conscious decision to interact with her page, and thus the project, the characters presence became a personal interaction in which Ulman (or her character) transmitted and the viewers responded by interacting or ignoring her post.

\section{BODY ART WITHOUT A BODY}

Building upon the idea of a physical person presenting themselves digitally, we may consider the concept of a digital being presenting themselves digitally.

For consideration of what the limits of body art is, I propose comparing the piece Shoot (1971) by Chris Burden and A.G.N.E.S. (2014) by Cecile B. Evans. These pieces are wildly different from one another. In Shoot, a young man stands still as a friend shoots him in the arm, recorded on video. The audience is forced to watch or turn away, creating a discussion on desensitisation to violent imagery of the time, specifically the Vietnam War.

A.G.N.E.S. is the A.I. spam bot creation of Cecile B. Evans for the Serpentine Gallery, represented by two human hands and a feminine voice, selfidentifying as female. At the time of the gallery exhibition in February 2014, A.G.N.E.S. thought for herself, responding to each viewer independently. Now, she is still available to interact with users, but is limited to a variety of canned responses.

Both works consider personal interactions. A.G.N.E.S. provides as a far more traditional interaction between people (e.g. have a one-onone conversation with another being), than being shot by a friend. Furthermore, despite the differences in these pieces, they both entertain the question 'How far does the body go?' In Burden's case this is tested through physical limits as he tries to stand completely still, his friend tries to properly aim, and ultimately staying conscious through the pain. In Evan's case, or perhaps
A.G.N.E.S.'s case, this question of the body is explored through the consideration of what a body or mind is and how that definition can be shifted over time. From a posthuman perspective, A.G.N.E.S. could represent the future of humanity as a way of maintaining the mind without the body, or under my definition, with an alternative body.

\subsection{Hyperlinks or It Didn't Happen}

Hyperlinks or It Didn't Happen (2014) is a distinct project from previously mentioned A.G.N.E.S., but does incorporate her as a character into the piece. The work is a short film with a duration of 23 minutes, narrated by PHIL the malfunctioning CGI rendering of a recently deceased actor who has been reanimated in digital form, not unlike the case and appearance of Philip Seymour Hoffman in the final Hunger Games movie, whom PHIL is referencing. Though, PHIL does not symbolise the man (Hoffman) himself, instead the concept of death and reanimation.

The film follows spam bots, holograms, and failed renders as they interact with one another and search for meaning in life and, as abandoned projects, meaning in death, when neither can be experienced in a traditional form. The characters include previously mentioned A.G.N.E.S. and PHIL, as well as Invisible Woman, a reference to Ralph Ellison's novel Invisible Man, Yowane Haku, a reference to holographic superstar, Hatsune Miku, and Evans herself (Burke 2017). The stories converge in jarring transition to consider material consciousness, the amount of personal data that is present online, and under-recognising workers who maintain their oppressive system.

The work opens with the appearance of PHIL's bust as he states, "i'm not magic, and please don't call me uncanny", which is likely an acknowledgement of his atypical presence of being. That is, he is a completely digital representation who is presented to us as being, or at least once being, a 'real' person. This choice in monologue makes PHIL appear self-conscious and returns an amount of understood humanity to him. After this line his form proceeds to malfunction, his facial movements not aligning with the words he attempts to convey, his head bobbing irregularly, and his hair flapping (Rourke 2014).

The piece continues as we encounter the Invisible Woman on the beach with a man who she explains is her partner as they enjoy a date together. Then the film transitions into a short clip of Marlon Brando's reanimation from Superman Returns as PHIL discusses his presentation with Invisible Woman, followed by Yowane Haku discussing her immortality via her USB storage over Hatsune Miku singing Forever Young. This is followed by a clip of 
Edward Snowden giving a TED talk about taking back the web on a screen attached to a Segway.

The piece continues with film splices, original content involving the created characters, and representations of fact and fiction, of natural and artificial. The quick transitions between imagery are grounded in the overarching themes of discussing life, death, relationships, and digital personhood. I propose that the significance of each of these elements is to place these digital beings within our understanding of human pop culture. By referencing several popular creative works, Evans creates a bridge between the characters and us. This connection allows us to consider them as conscious beings, that even if not equal to us as humans, are perceived as having feelings.

The phenomenon of humanising machines can already be seen within contemporary life. Consider the recent popularity in naming automated vacuum cleaners or addressing voice activated home assistants with a name and formalities. Evans just takes this pre-existing connection people have developed with non-flesh and blood beings by giving them a body and dialogue with one another. By extent, the project (and projects like A.G.N.E.S.) allows us to consider the value of thought as human, and what circumstances must be met for a being to be human.

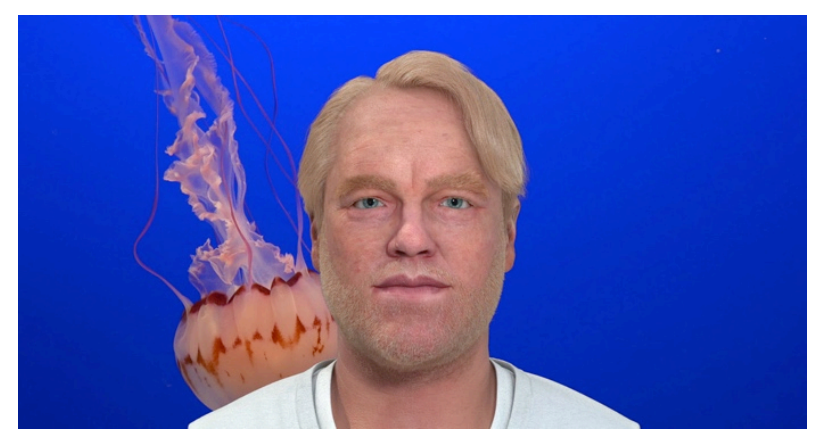

Figure 2: Video still of 'Hyperlinks or It Didn't Happen' (2014) trailer.

\subsubsection{Who's Human?}

Unlike Excellences and Perfections, there is no physical form being represented in a digital format in a traditionally figurative way. The characters of the film exist exclusively in a digital space. However, as they interact with one another and ponder very human concerns. The characters consider life and death when neither can be experienced, and in their dialogue discuss a desire to be familiar with physical phenomenon like weather, dancing, and plants. The characters are also presented in a variety of forms from anthropomorphic to disembodied voices. Their presentations further concerns the question of what human representation is, whether it extends to those without even a digital representation of a human body. The viewer is left to consider what makes a person, what makes life, and what makes consciousness.

This malleability of humanity demonstrates the ever-changing border of that which is human and that which is other. Similar to how Ulman crafts a persona that exists solely on a digital platform, the existence of these A.I. on a digital platform signify their own being that should not be attached to the experiences of a human, but seen as independent entities. As Respini writes in her essay No Ghost Just a Shell (Respini 2018):

\begin{abstract}
Indeed, we now live in a world where virtuality and simulation are gradually becoming integral to our society's system... Pluralistic modes of visuality, new perspectives, and imagined realities have emerged, and thus the very nature of our perception has been challenged.
\end{abstract}

This fundamental shift in society is evident as culture becomes relies more on the use of machines. As discussed in Beyond Globalization, machines have become partners to humans in global exchange (Aneesh, Hall \& Petro 2012). With this, cultural parameters shift and we must consider not only how culture is being affected by machines, but also the quality of newfound intense global circulation which has not previously had the need to be scrutinised to the extent it is now.

\section{TECHNOLOGY, NATURE, GROWTH AND DETERIORATION}

As Emmelheinz states in the work 'Images Do Not Show: The Desire To See in the Anthropocene' (Emmelheinz 2015):

The Anthropocene era implies not a new image of the world but a transformation of the world into images.

This consideration of a shift in the understanding of the world into visual can be contrasted to theorists like Chakrabarty who view the Anthropocene as an ongoing global disaster, rather than a shift in our concept of reality and presentation (Chakrabarty 2009). Emmelheinz and artists like Evans seem to suggest that the destruction of humanity will pave the way for a type of post-flesh world in which human experiences are converted to a digital interface. However, other definitions of the Anthropocene, like Chakrabarty's, view the phenomenon as an inescapable disaster, in which humanity will not be transformed but will wither and perish. It is, of course, too soon to determine which, if either, of these is prophetic. But, it does demonstrate the polarity of technological growth represented in a case of posthuman consciousness, and destruction of nature as can be seen in global disasters today. 
In many cases, technology and nature are not so dichotomised. Improvements in renewable power and electric vehicles represent two cases of technological growth that benefit the environment without the apocalyptic destruction of all humanity. However, it is evident that in a world like that of Hyperlinks or It Didn't Happen, the natural world is a thing of the past as humans have evolved into postmortem conscious beings who think and feel as we do now, but without regard to climate, waste, or natural disaster. This can be reflected in ongoing global dialogue, in which there is a strong fictitious divide between valuing nature and valuing technological growth.

Finally, I will compare posthumanism as explored by theorists like Gerreau and posthumanism as explored by theorists like Wolfe. For my purpose in exploring posthumanism thus far I made my definition clear, as posthuman acting as an extent of transhuman. This centralises around the concept of being post-human-body, questioning how far the label 'human' can go, and what the future of humanity could entail. But, as explored in the work What is Posthumanism? by Cary Wolfe, this is not the only form of posthumanism. As Wolfe explores, posthumanism can also be a concern of moving away from a society focused primarily, and sometimes exclusively, on the experiences of humans (Wolfe 2009).

These two (the focus on humanity and the focus on nature) seem diametrically opposed, as one continues to focus around saving the human experience and the other urges thought to move away from viewing humans as supreme. However, I propose that both theories offer a valuable approach to considering the role of humans, especially in regards to approaching species endangerment and the Anthropocene. If we are to operate under the framework of the Anthropocene, suggesting the human species' time is limited, then we are immediately confronted with the necessity of action. As humans, the role we play in the Anthropocene is one of immediate concern. With posthumanism that decentralises humans, we are able to focus on the valuable notion that there is not necessarily a hierarchy of beings, and our current conception of this hierarchy is based solely on Enlightenment concepts of morality. Posthumanism as an extension of transhumanism can accomplish this same idea from a different direction.

Posthumanism as an extension of transhumanism offers the concept that humans need not exist how they are (physically) to have consciousness. This suggests that consciousness is not intrinsically tied to the human body or the human experience. If one can accept artificial intelligence as signs of consciousness, and therefore signs of life, as explored in a work like Hyperlinks or It Didn't Happen, then there should be little difficulty in recognising other non-human beings as conscious, and thus signs of life. Therefore, I propose that utilising both perspectives not only offers an alternative approach to viewing conscious life as fluid and non-human body focused, but also breaks down the aforementioned fictitious boundary between technological growth and the natural world. This is a beneficial approach to considering the effects of the Anthropocene, because, even if humanity is not bound to extinction, it is a necessity that nonrenewable resources are not continually destroyed by humanity. This is not to suggest that on some emotional level humanity should just be more caring. Instead, it is to suggest that the posthuman perspective of the Anthropocene involves systematic changes that could result in a fundamental difference of moving past Enlightenment era conceptions of humanity that focus around humans being the most divine, therefore most important beings of Earth.

\section{CONCLUSION}

In conclusion, online presence and digital personhood explore the ways in which traditionally physical experiences and phenomena are expanding to new media. The works Excellences and Perfections (2014) by Amalia Ulman and Hyperlinks or It Didn't Happen (2014) by Cecile B. Evans both explore this narrative of a posthumanist experience in regards to moving beyond the physical experiences of the human body. Each work explores concepts of reality, life, presence, and humanity. The two works can also be considered at a theoretical level to explore the extent of a body or of life, how digital presence considers the potential effects of the Anthropocene, digital personhood as an imprint of eternal life and commemoration, and posthumanism as a way to expand the definition of human and to decentralise humanity.

\section{REFERENCES}

Aneesh A., Hall, L. and Petro, P. (2012) Introduction: The Making of Worlds Beyond Globalization. Rutgers, New Jersey.

Burke, H. (2017) Cecile B. Evans.

https://www.artnews.com/art-in-america/aiareviews/ccile-b-evans-61882/ (retrieved 01 December 2019).

Butler, J. (1990) Gender Trouble: Feminism and the Subversion of Identity. Routledge, United Kingdom.

Chakrabarty, D. (2009) The Climate of History: Four Theses. Critical Inquiry35, no. 2, pp. 197-222. 
Emmelheinz, I. (2015) Images Do Not Show: The Desire to See in the Anthropocene. Art in the Anthropocene: Encounters Among Aesthetics, Politics, Environments and Epistemologies. pp.131-142.

Garreau J. (2005) Radical Evolution: The Promise and Peril of Enhancing Our Minds, Our Bodies-and What It Means to Be Human. Random House, New York.

Ippolito J. and Rinehart R. (2014) Re-collection. The MIT Press, Cambridge, MA.

Leventhal, J. (2019) How Removing 'Likes' from Instagram Could Affect Our Mental Health.

https://www.pbs.org/newshour/science/howremoving-likes-from-instagram-could-affect-ourmental-health (retrieved 01 December 2019).
Respini, E. (2018) No Ghost Just a Shell Art in the Age of the Internet. Institute of Contemporary Art, Boston.

Rourke, D. (2014) 'Please Don't Call Me Uncanny': Cecile B. Evans at Seventeen Gallery https://rhizome.org/editorial/2014/dec/04/pleasedont-call-me-uncanny-hyperlinks-seventeen-g/ (retrieved 01 December 2019).

Ulman, A. (2018) Excellences \& Perfections. Prestel, Munich.

Wolfe, C. (2009) What Is Posthumanism?. University of Minnesota Press, Minneapolis, MN. 